\title{
A murine model of inflammation-induced cerebral microbleeds
}

\author{
Rachita K. Sumbria ${ }^{1,2}$, Mher Mahoney Grigoryan², Vitaly Vasilevko ${ }^{3}$, Tatiana B. Krasieva ${ }^{4}$, Miriam Scadeng ${ }^{5}$, \\ Alexandra K. Dvornikova², Annlia Paganini-Hill ${ }^{2}$, Ronald Kim ${ }^{6}$, David H. Cribbs ${ }^{3}$ and Mark J. Fisher $2,6,7,8^{*}$
}

\begin{abstract}
Background: Cerebral microhemorrhages $(\mathrm{CMH})$ are tiny deposits of blood degradation products in the brain and are pathological substrates of cerebral microbleeds. The existing $\mathrm{CMH}$ animal models are $\beta$-amyloid-, hypoxic brain injury-, or hypertension-induced. Recent evidence shows that CMH develop independently of hypoxic brain injury, hypertension, or amyloid deposition and $\mathrm{CMH}$ are associated with normal aging, sepsis, and neurodegenerative conditions. One common factor among the above pathologies is inflammation, and recent clinical studies show a link between systemic inflammation and $\mathrm{CMH}$. Hence, we hypothesize that inflammation induces $\mathrm{CMH}$ development and thus, lipopolysaccharide (LPS)-induced CMH may be an appropriate model to study cerebral microbleeds.
\end{abstract}

Methods: Adult C57BL/6 mice were injected with LPS (3 or $1 \mathrm{mg} / \mathrm{kg}$, i.p.) or saline at 0, 6, and $24 \mathrm{~h}$. At 2 or 7 days after the first injection, brains were harvested. Hematoxylin and eosin (H\&E) and Prussian blue (PB) were used to stain fresh (acute) hemorrhages and hemosiderin (sub-acute) hemorrhages, respectively. Brain tissue ICAM-1, IgG, Iba1, and GFAP immunohistochemistry were used to examine endothelium activation, blood-brain barrier (BBB) disruption, and neuroinflammation. MRI and fluorescence microscopy were used to further confirm $\mathrm{CMH}$ development in this model.

Results: LPS-treated mice developed H\&E-positive (at 2 days) and PB-positive (at 7 days) CMH. No surface and negligible H\&E-positive CMH were observed in saline-treated mice $(n=12)$. LPS (3 mg/kg; $n=10)$ produced significantly higher number, size, and area of H\&E-positive CMH at 2 days. LPS $(1 \mathrm{mg} / \mathrm{kg} ; n=9)$ produced robust development of PB-positive $\mathrm{CMH}$ at 7 days, with significantly higher number and area compared with saline ( $n=9)$-treated mice. CMH showed the highest distribution in the cerebellum followed by the sub-cortex and cortex. LPS-induced CMH were predominantly adjacent to cerebral capillaries, and CMH load was associated with indices of brain endothelium activation, BBB disruption, and neuroinflammation. Fluorescence microscopy confirmed the extravasation of red blood cells into the brain parenchyma, and MRI demonstrated the presence of cerebral microbleeds.

Conclusions: LPS produced rapid and robust development of H\&E-positive (at 2 days) and PB-positive (at 7 days) CMH. The ease of development of both H\&E- and PB-positive CMH makes the LPS-induced mouse model suitable to study inflammation-induced $\mathrm{CMH}$.

Keywords: Animal models, Cerebral microhemorrhage, Cerebral microbleeds, Inflammation, Hemosiderin

Abbreviations: $\mathrm{CMH}$, Cerebral microhemorrhages; LPS, Lipopolysaccharide; H\&E, Hematoxylin and eosin; PB, Prussian blue; MRI, Magnetic resonance imaging; CAA, Cerebral amyloid angiopathy; COPD, Chronic obstructive pulmonary disease; TBI, Traumatic brain injury; DAB, 3,3'-Diaminobenzidine; TPEF, Two-photon-excited fluorescence; BBB, Bloodbrain barrier

\footnotetext{
* Correspondence: mfisher@uci.edu

${ }^{2}$ Department of Neurology, University of California, Irvine, CA, USA

${ }^{6}$ Department of Pathology and Laboratory Medicine, University of California,

Irvine, CA, USA

Full list of author information is available at the end of the article
} 


\section{Background}

Cerebral microhemorrhages $(\mathrm{CMH})$ are tiny perivascular deposits of blood degradation products in the brain and are the pathological substrate of cerebral microbleeds [1]. In spite of the significant clinical and scientific interest in this field, lack of appropriate animal models has hindered progress in delineating the exact mechanisms involved in $\mathrm{CMH}$ development and in the development of treatments to address $\mathrm{CMH}$. The currently used animal models of $\mathrm{CMH}$ are amyloid beta $(\mathrm{A} \beta)-[2-4]$, hypoxia-reoxygenation-, or hypertension-induced [5]. These existing animal models have several disadvantages: (1) CMH development in these models can take up to 15-24 months, (2) invasive surgical procedures are required to exacerbate $\mathrm{CMH}$ development, and most importantly, (3) clinically, $\mathrm{CMH}$ may develop independent of amyloid deposition, hypoxic brain injury, or hypertension [6].

It is now well recognized that $\mathrm{CMH}$ are not only associated with cerebrovascular diseases including stroke, cerebral amyloid angiopathy (CAA), and cerebral hypertensive vasculopathy [1] but are also found in patients with sepsis [7], Parkinson's disease [8], chronic obstructive pulmonary disease (COPD) [9], and traumatic brain injury (TBI) [10] and in normal aging adults [1]. One common feature of these entities is systemic inflammation [11], and recent human studies show a link between systemic inflammation and $\mathrm{CMH}$ pathogenesis. Higher levels of peripheral inflammatory markers are associated with cerebral microbleeds in aging patients [12], high levels of circulating tumor necrosis factor receptor 2 are observed in subjects with cerebral microbleeds [13], and higher activity of lipoprotein phospholipaseA2 (a marker of vascular inflammation) is related to the presence of deep cerebral microbleeds in subjects who were carriers of at least one APOE $\varepsilon 2$ or $\varepsilon 4$ allele [14]. While inflammation may be central to the development of $\mathrm{CMH}$, a direct causal link between inflammation and $\mathrm{CMH}$ development has been lacking. Hence, we hypothesized that systemic inflammation will induce $\mathrm{CMH}$ development and that an inflammationinduced animal model will be appropriate to study $\mathrm{CMH}$ development and treatment.

To test this hypothesis, we used lipopolysaccharide (LPS), a well-characterized standardized inflammatory stimulus, to study CMH development. Previous studies from our lab showed that mice treated with a $5 \mathrm{mg} / \mathrm{kg}$ dose of LPS at 0 and $24 \mathrm{~h}$ had a significantly higher number of $\mathrm{CMH}$ at 2 days as evident by hematoxylin and eosin (H\&E)-positive staining in brain tissue compared with controls. We now report a well-characterized inflammation-induced mouse model of $\mathrm{CMH}$ with low mortality, using different dosing regimens $(1$ or $3 \mathrm{mg} / \mathrm{kg}$, i.p., at 0,6 , and $24 \mathrm{~h}$, and sacrifice at 2 or 7 days) of LPS.
In the current study, we examined both acute $\mathrm{CMH}$ (H\&E-positive) at 2 days and sub-acute CMH (Prussian blue/hemosiderin-positive) at 7 days. The number, size, total area, and neuroanatomical distribution of $\mathrm{CMH}$ were examined in the LPS- and saline-treated mice. To elucidate mechanisms involved in LPS-induced $\mathrm{CMH}$, we analyzed markers of brain endothelial damage (ICAM-1 and parenchymal IgG) and neuroinflammation (astrocyte and microglia/macrophages). The vascular source of LPS-induced CMH was examined, and we also used fluorescence microscopy to confirm acute $\mathrm{CMH}$ development and magnetic resonance imaging (MRI) to confirm the radiographic presence of cerebral microbleeds.

\section{Methods}

\section{Mouse treatment}

All animal procedures were approved by the UCI Institutional Animal Care and Use Committee and were carried out in compliance with the University Laboratory Animal Resources regulations. Adult (male and female 10-12 weeks old) C57BL/6 mice (Taconic, Hudson, NY) were used for all the experiments. In the first set of experiments, the mice were treated with either a $3 \mathrm{mg} / \mathrm{kg}$ dose of LPS derived from Salmonella typhimurium (Sigma, St. Louis, MO) or saline i.p. at 0, 6, and $24 \mathrm{~h}$ and sacrificed 2 days after the first injection to examine acute $\mathrm{CMH}$ development. In a separate series of experiments, the mice were treated with a $1 \mathrm{mg} / \mathrm{kg}$ dose of LPS or saline at 0,6 , and $24 \mathrm{~h}$ and sacrificed 7 days after the first injection to examine sub-acute $\mathrm{CMH}$ development. The mice fed and drank ad lib and received up to three times daily doses of $1 \mathrm{~cm}^{3}$ saline subcutaneously. Two or 7 days after the first injection, mice were anesthetized with a lethal dose of Nembutal $(150 \mathrm{mg} / \mathrm{kg}$, i.p.), cardiac perfusions were performed using ice-cold PBS for $5 \mathrm{~min}$ to clear the cerebral vasculature, and brains were processed for $\mathrm{CMH}$ detection.

\section{Microhemorrhage detection}

Brains were fixed in $4 \%$ paraformaldehyde at $4{ }^{\circ} \mathrm{C}$ for $72 \mathrm{~h}$, examined for surface microhemorrhages, and sectioned into $40-\mu \mathrm{m}$ coronal sections using a vibratome (Technical Products International, Inc., St. Louis, MO). Every sixth section was collected and stained with either H\&E to detect fresh (acute) microhemorrhages in the 2day study or Prussian blue (PB) to detect hemosiderin (a marker of sub-acute microhemorrhage) in the 7-day study. PB was not used for the 2-day study, based on findings of our earlier work [15]. A total of approximately 30 brain sections were analyzed per mouse. For PB staining, sections were stained using freshly made $5 \%$ potassium hexacyanoferrate trihydrate (Sigma, St. Louis, MO) and 5 \% hydrochloric acid (Sigma, St. Louis, 
MO) for $30 \mathrm{~min}$, rinsed in water and counterstained with Nuclear Fast Red (Sigma, St. Louis, MO), dehydrated, and cover-slipped. H\&E staining was performed by Research Services Core offered by the Department of Pathology and Laboratory Medicine at the UCI Medical Center. $\mathrm{CMH}$ were counted at a $\times 20$ magnification by a blinded observer as a collection of red blood cells (RBC) that appear red-orange using H\&E stain and as clear purple-blue deposits using $\mathrm{PB}$. Digitized images were obtained using an Olympus BX40 microscope and CC12 Soft-Imaging System with Olympus MicroSuite (TM)-B3SV software. $\mathrm{CMH}$ size $\left(\mu \mathrm{m}^{2}\right)$ and positive area (expressed as a percent of total area analyzed) were determined by an observer blinded to the experiment using the NIH ImageJ software 1.62. When the vessel associated with the $\mathrm{CMH}$ was visible, internal diameter of the blood vessel was determined using the NIH ImageJ software 1.62 .

\section{ICAM-1, IgG, Iba1, and GFAP immunohistochemistry}

To determine the role of endothelial damage and neuroinflammation in LPS-induced $\mathrm{CMH}$ development, ICAM-1 (marker of endothelial cell activation), parenchymal IgG (blood-brain barrier (BBB) damage marker), Iba1 (microglia/macrophage marker), and GFAP (astrocyte marker) immunohistochemistry were performed. Briefly, $40-\mu \mathrm{m}$ sections from mice treated with LPS and saline from the 2-day study were incubated in $0.5 \%$ hydrogen peroxide in $0.1 \mathrm{M}$ PBS $(\mathrm{pH}$ 7.4) containing $0.3 \%$ Triton-X100 (PBST) for $30 \mathrm{~min}$ at room temperature to block endogenous peroxidase activity. After washing with PBST, sections were incubated for $30 \mathrm{~min}$ with PBST containing $2 \%$ bovine serum albumin to block non-specific protein binding. Sections were then incubated overnight at $4{ }^{\circ} \mathrm{C}$ with a rabbit anti-mouse IgG antibody (1:200 dilution; Jackson ImmunoResearch, West Grove, PA), rabbit monoclonal antibody against ICAM-1 (1:500 dilution; Abcam, Cambridge, MA), rabbit antibody against Iba1 (1:200 dilution, Wako Chemicals USA, Richmond, VA), or rabbit antibody against GFAP (1:2000 dilution, Abcam, Cambridge, MA). After washing with PBST, sections were incubated at room temperature for $1 \mathrm{~h}$ with biotinylated anti-rabbit IgG (1:500 dilution; Jackson ImmunoResearch, West Grove, PA), followed by 1-h incubation at room temperature with $\mathrm{ABC}$ complex according to the manufacturer instructions (Vector Laboratories, Burlingame, CA). Sections were developed with 3,3'-diaminobenzidine (DAB) (Vector Laboratories, Burlingame, CA). Sixteen images per brain section were acquired at $\times 20$ magnification, and the total positive immunoreactive area was quantified using the NIH ImageJ software by an observer blinded to the experimental groups.
Immunopositive area was expressed as percent of total area analyzed.

\section{Magnetic resonance imaging}

MRI was performed to confirm the radiographic presence of cerebral microbleeds in this model using a randomly selected subset of mouse brains after completion of the 7-day experiment. Mouse brains were collected post mortem after cardiac perfusion with PBS to clear vasculature of blood, followed by fixation in $4 \%$ paraformaldehyde; imaging was performed prior to sectioning. Data were acquired using a Bruker $7 \mathrm{~T}$ small-animal MRI machine with a $12-\mathrm{cm}$ gradient, a $660 \mathrm{~m} / \mathrm{T} / \mathrm{m}$ strength and 4570 slew rate, and a $1-\mathrm{cm}$ receive-only surface coil. The pulse sequence used was a 3D FLASH (fast low-angle shot) gradient echo sequence. TE was $12 \mathrm{~ms}$, TR was $30.2 \mathrm{~ms}$, and FA was $11^{\circ}$. Voxel size was $100 \times 100 \times 156 \mu \mathrm{m}$. The MRI data was manually processed and surface-rendered using Amira software (FEI, Hillsboro, OR).

\section{Ex vivo optical microscopy imaging}

In a separate series of experiments, the transit of $R B C$ across the cerebral vasculature into the brain parenchyma was visualized using ex vivo confocal and twophoton-excited fluorescence (TPEF) microscopy. Briefly, autologous blood was collected from inbred Tie2-GFP mice (Jackson Laboratory, Bar Harbor, ME) in which endothelial cells are labeled with green fluorescent protein (GFP), and RBC were purified using Ficoll-Paque (GE Healthcare, Uppsala, Sweden) gradient. After several washes in PBS, RBC were stained with PKH26 Red Fluorescent Cell Linker Kit (Sigma, St. Louis, MO) according to the manufacturer's instructions and reinjected into the mice. Immediately after RBC injection, the mice were subjected to either saline or LPS tripledosing regimen $(3 \mathrm{mg} / \mathrm{kg}$, i.p., 0,6 , and $24 \mathrm{~h})$ as per the 2-day experiment described earlier. The mice were sacrificed $48 \mathrm{~h}$ after the first saline or LPS injection, and whole brains were collected and fixed in $4 \%$ paraformaldehyde for imaging. Fluorescence and second-harmonic generation (SHG) images of whole brains fixed in $4 \%$ paraformaldehyde were obtained using Zeiss LSM 510 Meta NLO microscopy system equipped with a long working distance Zeiss $40 \times 0.8$ water immersion objective. GFP fluorescence was excited by $488-\mathrm{nm}$ line of the Argon laser; PKH26 excitation was provided by $\mathrm{He}-\mathrm{Ne}$ 543-nm laser, and SHG signal from collagen was generated using a Chameleon Ultra femtosecond pulsed tunable laser (Coherent Inc., Los Angeles, CA) at $800 \mathrm{~nm}$. Two confocal fluorescence channels (green emission at $500-530 \mathrm{~nm}$ and red emission at 565-615 nm) were acquired simultaneously, and SHG image (blue emission filter 390-465 nm) was acquired consequently. Laser 
scanning did not induce any visible damage to the cells or noticeable bleaching of the sample. Stacks of images were acquired with the $z$-step (distance between consecutive imaging planes) of $2.5 \mu \mathrm{m}$. The maximum depth for imaging was up to $80 \mu \mathrm{m}$ from the brain surface. The probed 3D volume was reconstructed by Zeiss LSM original software.

\section{Statistical analysis}

Data were represented as mean \pm SEM, and all statistical analysis was performed using GraphPad Prism 5 (GraphPad Software Inc., La Jolla, CA). Student's $t$ test (for normally distributed data) or Mann-Whitney $U$ test (for non-normal data) was used to compare two groups. One- and two-way ANOVA (with and without repeated measures) with Bonferroni's post hoc test were used to compare more than two groups. Spearman's rho correlation was used for correlation analysis. A $p$ value of $<0.05$ was considered statistically significant.

\section{Results}

Survival

For the 2-day experiment, all 12 mice in the saline group survived the duration of the study; however, one mouse in the LPS group died before the end of the study, and two were excluded due to incomplete perfusion, giving us a total of ten mice in the LPS group. All mice $(n=9$ per group) survived the 7-day experiment.

\section{Surface cerebral microhemorrhages}

In the 2-day experiment, a $3 \mathrm{mg} / \mathrm{kg}$ dose of LPS at 0,6 , and $24 \mathrm{~h}$ induced the development of grossly visible surface $\mathrm{CMH}(7.4 \pm 2.2$ per brain), compared with saline controls that exhibited no surface $\mathrm{CMH} \quad(p<0.01)$ (Fig. 1a-c). Regional differences were observed in the location of these surface CMH. In the LPS-treated group, the average number of surface $\mathrm{CMH}$ per brain was significantly higher in the cerebellum/brain stem (4.7 \pm 1.6$)$ compared with the olfactory bulb $(1.8 \pm 0.6, p$ $<0.05)$ and the cerebral cortex $(0.9 \pm 0.3, p<0.01)$ (Fig. 1a). In mice treated with a $1 \mathrm{mg} / \mathrm{kg}$ dose of LPS at 0,6 , and $24 \mathrm{~h}$ and sacrificed 7 days after the first injection, the average number of surface $\mathrm{CMH}(1.2 \pm 0.4$ per brain) was significantly higher compared with the saline-treated mice, which again exhibited no surface CMH $(p<0.05$; Fig. $1 d)$.

\section{Acute parenchymal cerebral microhemorrhages}

In the 2-day experiment, a $3 \mathrm{mg} / \mathrm{kg}$ dose of LPS at 0,6 , and $24 \mathrm{~h}$ significantly induced $(p<0.0001$ vs saline control) the formation of fresh H\&E-positive parenchymal $\mathrm{CMH}(1.3 \pm 0.3$ vs $0.03 \pm 0.02$ per brain section). H\&Epositive $\mathrm{CMH}$ were observed in all LPS-treated mice (ranging from 0.4 to 2.9 per brain section). Regional differences were observed in the distribution of H\&Epositive $\mathrm{CMH}$ in the LPS-treated mice (Fig. 2a, b). The number of $\mathrm{H} \& \mathrm{E}$-positive $\mathrm{CMH}$ was the highest in the cerebellum/brain stem $(1.6 \pm 0.4$ per brain section $)$ followed by the sub-cortical $(0.8 \pm 0.2$ per brain section, $p<0.0001$ vs cerebellum and $p<0.05$ vs cortex) and the cortical $(0.4 \pm 0.09$ per brain section, $p<0.0001$ vs cerebellum) regions of the brains of LPS-treated mice. No regional differences were observed in the distribution of H\&E-positive CMH in the saline-treated mice (cortex $0.01 \pm 0.007$, sub-cortex $0.01 \pm 0.007$, and cerebellum/ brain stem $0.05 \pm 0.04$ per brain section). H\&E-positive $\mathrm{CMH}$ were also examined in the 7-day study to see if acute $\mathrm{CMH}$ continued to develop after LPS injections were stopped at 2 days. No significant differences in the number of H\&E-positive CMH were found between the LPS- and saline-treated mice in the 7-day study (data not shown).

A total of 68 blood vessels associated with H\&Epositive $\mathrm{CMH}$ were visible and studied in the current study. Of these, 51 were $<10 \mu \mathrm{m}$ in diameter (mean diameter $5.6 \pm 0.04 \mu \mathrm{m}$ ) and 17 were between 10 and $30 \mu \mathrm{m}$ in diameter (mean diameter $15.8 \pm 1.7 \mu \mathrm{m}$ ). The mean diameter of the vessels associated with the H\&Epositive CMH was $8.2 \pm 0.09 \mu \mathrm{m}$ (Fig. 2c), and the blood vessels ranged from 1.5 to $30 \mu \mathrm{m}$ in diameter.

Approximately 30 sections per mouse brain were examined in the current study. To study the size distribution of the parenchymal $\mathrm{CMH}$, all observed $\mathrm{CMH}$ from all the mice in each group were studied. A total of 616 (from $n=10$ ) and 9 (from $n=12$ ) H\&E-positive $\mathrm{CMH}$ were analyzed in the LPS- and saline-treated groups, respectively. H\&E-positive $\mathrm{CMH}$ ranged from 136 to $1684 \mu \mathrm{m}^{2}$ in the saline group and from 63 to $3.9 \times 10^{6} \mu \mathrm{m}^{2}$ in the LPS group (Fig. 3a). LPS produced $\mathrm{H} \& \mathrm{E}$-positive $\mathrm{CMH}$ that were significantly larger in size compared with saline controls $(p<0.0001)$. The mean size of H\&E-positive CMH was $2649 \pm 306$ and $246 \pm 145 \mu^{2}$ in the LPS- and saline-treated mice, respectively (Fig. 3b). Total H\&E-positive CMH area (or total $\mathrm{CMH}$ load, is a function of both the number and size of microhemorrhages) was significantly higher $(p<0.0001)$ in the LPS- compared with the salinetreated group $\left(0.01 \pm 0.004 \%\right.$ vs $4.8 \times 10^{-5} \pm 2.7 \times$ $10^{-5} \%$ of total brain section analyzed area) (Fig. 3c). The weights of the mice at the beginning and end of the 2-day study were $21.8 \pm 0.9$ and $21.3 \pm 0.8 \mathrm{~g}$ in the LPS group and $21.9 \pm 0.7$ and $22.6 \pm 0.76 \mathrm{~g}$ in the saline group, respectively.

\section{Sub-acute parenchymal cerebral microhemorrhages}

PB-positive stains were detected in both mice treated with saline and $1 \mathrm{mg} / \mathrm{kg}$ dose of LPS at 0,6 , and $24 \mathrm{~h}$ and sacrificed at 7 days. However, the mean number of PB-positive stains was significantly higher $(p<0.0001)$ in 


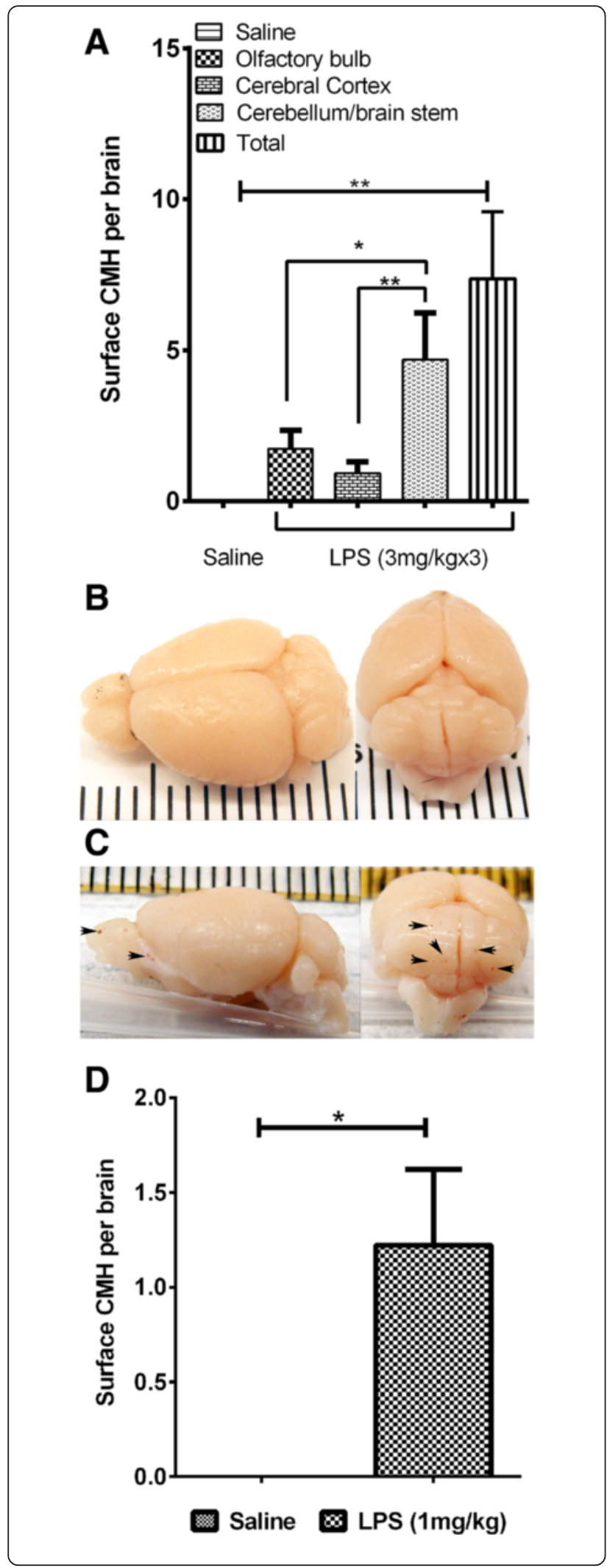

Fig. 1 Significant development of surface microhemorrhages in mice treated with LPS $(n=10)(3 \mathrm{mg} / \mathrm{kg}$ at 0,6 , and $24 \mathrm{~h})$ compared with saline-treated mice $(n=12)$ and regional differences in the distribution of surface microhemorrhages in LPS-treated mice (a) in the 2-day experiment. Brain images showing absence of surface microhemorrhages in saline-treated mice (b) and presence of surface microhemorrhages in the olfactory bulb (left panel) and the cerebellum (right panel) of LPS-treated mice (c). Significantly higher surface microhemorrhages in mice treated with LPS $(n=9)(1 \mathrm{mg} / \mathrm{kg}$ at 0,6 , and 24 h) compared with the saline-treated mice $(n=9)$ in the 7-day experiment (d). Data are presented as mean \pm SEM. Student's $t$ test for two groups and one-way ANOVA with Bonferroni's post-test for more than two groups; ${ }^{*} p<0.05,{ }^{* *} p<0.01$

the LPS-treated group $(1.9 \pm 0.2$ per brain section) compared with saline control $(0.8 \pm 0.1$ per brain section $)$ (Fig. 4a). Similar to H\&E-positive fresh CMH, PB-positive stains showed differences in regional distribution among the LPS-treated mice (Fig. 4b, c). The number of PB-positive stains was significantly higher in the cerebellum/brain stem $(1.9 \pm 0.3$ per brain section) compared with the cortical $(1.0 \pm 0.1$ per brain section, $p<$ $0.0001)$ and the sub-cortical $(0.9 \pm 0.1$ per brain section, $p<0.0001)$ regions of the brains of LPS-treated mice. No regional differences were observed in the distribution of PB-positive stains in the saline-treated mice (cortex $0.6 \pm 0.09$, sub-cortex $0.3 \pm 0.08$, and cerebellum/brain stem $0.5 \pm 0.1$ per brain section). A total of 466 (from $n=9$ ) and 231 (from $n=9$ ) PBpositive stains were analyzed in the LPS- and salinetreated groups, respectively. PB-positive stains ranged from 9 to $29,377 \mu \mathrm{m}^{2}$ among the LPS mice and from 14 to $4888 \mu^{2}$ among the saline-treated mice. The average PB-positive stain size was not significantly different in the LPS- and saline-treated mice (data not shown). The total PB-positive area was significantly $(p<0.01)$ higher in the LPS-treated mice $(0.002 \pm 0.0006 \%$ of total brain section area analyzed) compared with saline controls $(0.0006 \pm 0.0001 \%$ of total brain section area analyzed) (Fig. 4c). For the 7day study, the average weights of the mice at the beginning and end of the study were $21.0 \pm 0.6$ and $22.1 \pm 0.7 \mathrm{~g}$ among the saline mice and $20.7 \pm 1$ and $20.6 \pm 0.7 \mathrm{~g}$ among the LPS mice.

\section{Correlation between CMH development and brain endothelial damage}

Immunohistochemical analysis using sections (eight mice from the LPS-treated group and four mice from the saline-treated group) from the 2-day experiment revealed that ICAM-1-positive area was significantly associated with the number of $\mathrm{CMH}$ per brain section $(r=0.86, p<0.001)$ and total CMH-positive area (or $\mathrm{CMH}$ load $)$ expressed as \% total area $(r=0.70, p<0.01)$. 


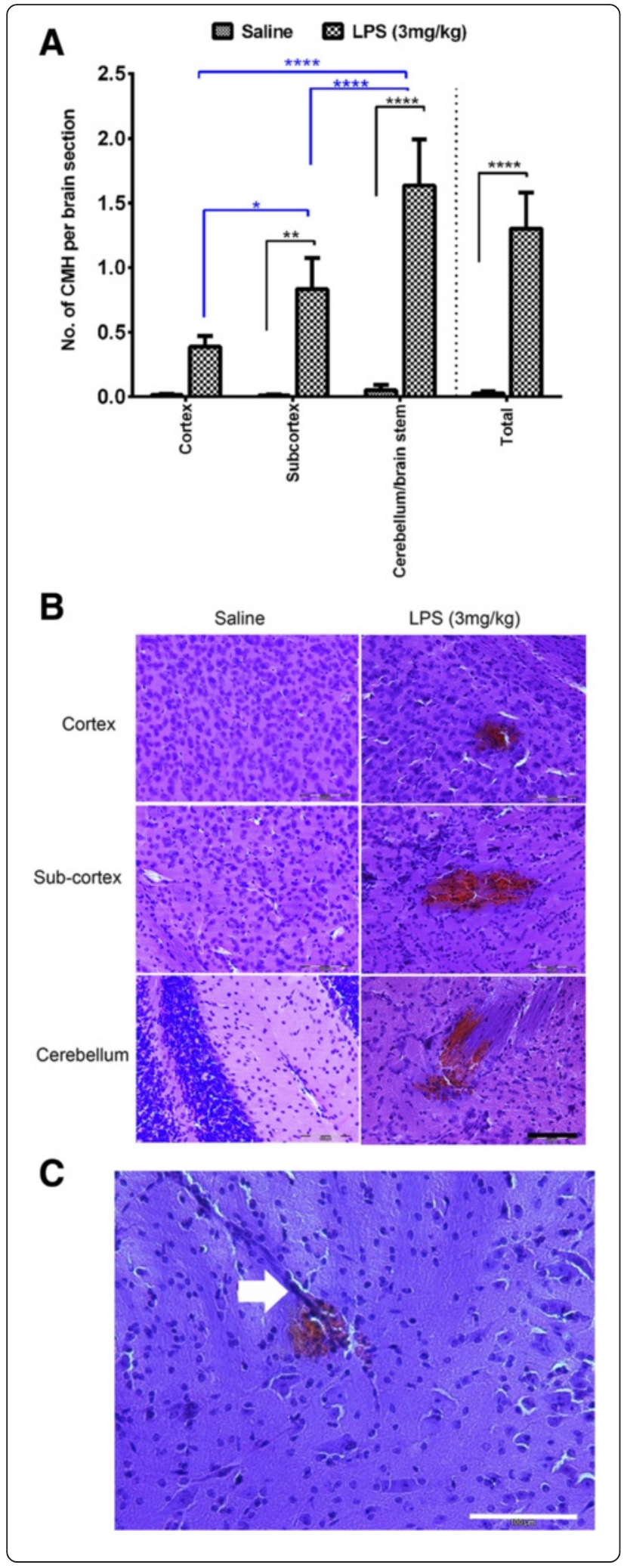

Fig. 2 Acute H\&E-positive cerebral microhemorrhages: Significant development of acute (H\&E-stained) parenchymal microhemorrhages in mice treated with LPS $(n=10)(3 \mathrm{mg} / \mathrm{kg}$, at 0,6 , and $24 \mathrm{~h})$ compared with saline-treated mice $(n=12)$ (comparisons shown in black) (a). Significant difference in regional distribution of H\&E-positive parenchymal microhemorrhages in the LPS-treated group (comparisons shown in blue) (a). Representative images showing H\&E-positive cerebral microhemorrhages in the cortex, sub-cortex, and cerebellum of LPS- and saline-treated mice (b). Representative image showing a cerebral blood vessel associated with an acute (H\&E-positive) cerebral microhemorrhage (c). Data are presented as mean \pm SEM. Two-way repeated measure ANOVA with Bonferroni's post-test; ${ }^{*} p<0.05,{ }^{* *} p<0.01$, ${ }^{* * *} p<0.0001$. Scale bar $=100 \mu \mathrm{m}$

IgG-positive area was significantly associated with $\mathrm{CMH}$ size $(r=0.56, p<0.05)$ and total $\mathrm{CMH}$-positive area (or CMH load) $(r=0.53, p<0.05)$, as shown in Table 1.

\section{Correlation between CMH development and neuroinflammation}

Immunohistochemical analysis using brain sections (seven mice from the LPS-treated group and four mice from the saline-treated group) from the 2-day experiment showed that the total Iba1- and GFAP (markers of neuroinflammation)-positive immunoreactive areas were significantly higher in the LPS-treated mice compared with the saline controls (Fig. 5a-c). Further, both Iba1and GFAP-positive immunoreactive areas were significantly associated with the number (Iba1 $r=0.85, p<$ 0.01 ; GFAP $r=0.83, p<0.01$; Fig. $5 \mathrm{~d}$ ), size (Iba1 $r=0.72$, $p<0.01$; GFAP $r=0.62, p<0.05$ ), and total $\mathrm{CMH}$ load (Iba1 $r=0.88, p<0.001$; GFAP $r=0.85, p<0.001$ ), as shown in Table 1.

Apart from histochemistry, in the current model, the development of parenchymal $\mathrm{CMH}$ and cerebral microbleeds was further confirmed by two separate methodologies. In the 2-day experiment, ex vivo confocal fluorescence microscopy showed the transit of fluorescently labeled RBC (PKH26 labeled red RBC; Fig. 6a, b) across the cerebral vascular endothelium (GFP labeled green endothelium; Fig. 6a, b) into the brain parenchyma in the LPS-treated mice (Fig. 6b) but not the salinetreated mice (Fig. 6a). In the 7-day experiment, MRI confirmed robust development of cerebral microbleeds in the LPS-treated mice (Fig. 6c).

\section{Discussion}

In the current study, we have developed and characterized an LPS-induced inflammation mouse model of $\mathrm{CMH}$ that shows rapid and robust development of $\mathrm{CMH}$. As early as 2 days following an intraperitoneal LPS triple-dosing regimen $(3 \mathrm{mg} / \mathrm{kg}$ at 0,6 , and $24 \mathrm{~h}$ ), $\mathrm{H} \& \mathrm{E}$-positive (acute) $\mathrm{CMH}$ were observed in mice. $\mathrm{PB}$ positive $\mathrm{CMH}$ were observed in mice at 7 days following 


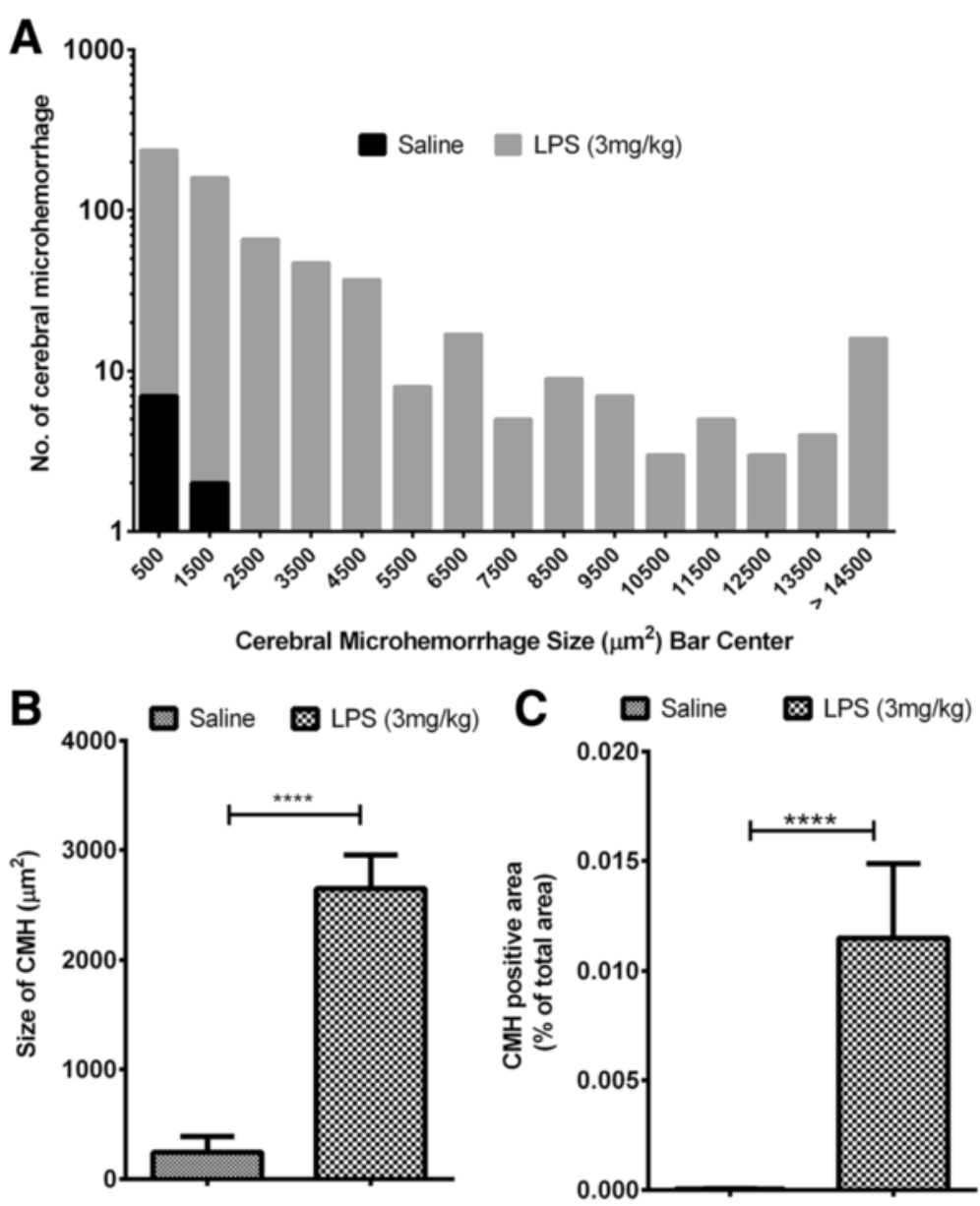

Fig. 3 Size and area of acute H\&E-positive CMH: Size distribution of all the H\&E-positive CMH analyzed in the LPS $(n=10)(3 \mathrm{mg} / \mathrm{kg}$, at 0, 6, and $24 \mathrm{~h}$ )- and saline $(n=12)$-treated mice (a). The width of each bar is $1000 \mathrm{\mu m}^{2}$, and the bin center for each bar is shown on the X-axis. H\&Estained cerebral microhemorrhage average size (b) and positive area (c) are significantly higher in LPS-treated (3 mg/kg, at 0, 6, and 24 h) mice compared with saline controls. Data are presented as mean \pm SEM. Mann-Whitney $U$ test to compare two groups; ${ }^{* * *} p<0.0001$

a modified intraperitoneal LPS triple-dosing regimen $(1 \mathrm{mg} / \mathrm{kg}$ at 0,6 , and $24 \mathrm{~h})$. Both the H\&E- and PBpositive lesions showed similar neuroanatomical distribution patterns, with the highest number of $\mathrm{CMH}$ located in the cerebellum followed by the sub-cortex and cortex. The total CMH load was significantly associated with markers of cellular activation (endothelium, astrocyte, and microglia/macrophage) and BBB disruption. Finally, MRI confirmed the presence of cerebral microbleeds in this LPS-induced inflammation mouse model.

Systemic inflammation and an increase in inflammatory markers have been associated with normal aging [11], stroke [16-18], and peripheral conditions including sepsis [19] and hypertension [20]. Active inflammation can cause endothelial damage and BBB disruption [21], and it is speculated that these events allow extravasation of erythrocytes from the blood vessel lumen into the brain parenchyma, resulting in $\mathrm{CMH}$ development [22]. In fact, recent studies show an association between active inflammation and $\mathrm{CMH}[12,13]$. Inflammation may thus produce vascular dysfunction that triggers or potentiates $\mathrm{CMH}$ development.

LPS is a well-studied inflammatory stimuli used in rodent models of acute inflammation [23] that causes BBB damage [24] and brain endothelial dysfunction $[25,26]$. These characteristics of LPS make it a wellsuited stimulus for the development of inflammationinduced $\mathrm{CMH}$. We have previously shown that two intraperitoneal injections of LPS $(5 \mathrm{mg} / \mathrm{kg}$ at 0 and $24 \mathrm{~h}$ ) result in the development of H\&E-positive $\mathrm{CMH}$ in $\mathrm{C} 57 \mathrm{BL} / 6$ mice, at 2 days after the first LPS injection [15]. In the current study, we used a modified dosing regimen, adapted from a published study [27] and found that triple intraperitoneal injections of LPS $(3 \mathrm{mg} / \mathrm{kg}$ at 0,6 , and $24 \mathrm{~h}$ ) result in rapid (as early as 2 days) development of H\&E-positive CMH, with negligible mortality. 


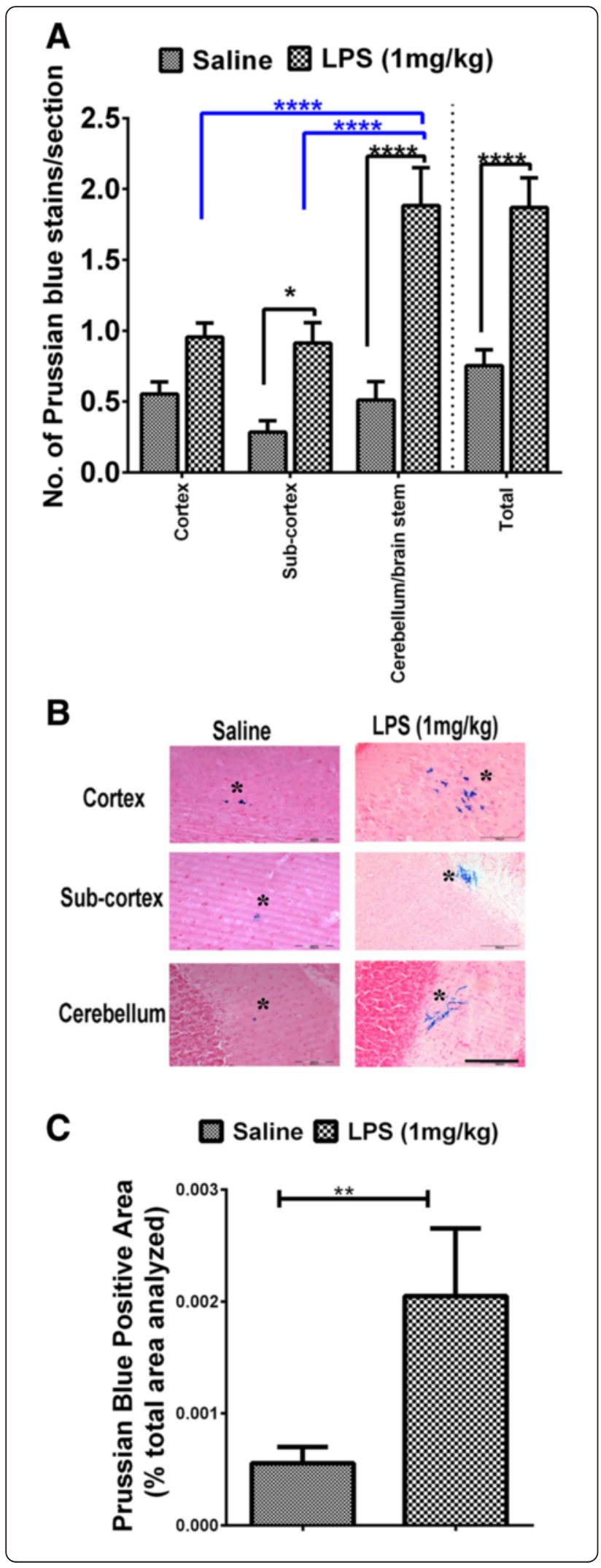

Fig. 4 Sub-acute Prussian blue-positive cerebral microhemorrhages: Significantly higher number of PB-positive stains in LPS $(1 \mathrm{mg} / \mathrm{kg}$, at $0,6$, and 24 h; $n=9)$ - compared with saline-treated $(n=9)$ mice (comparisons shown in black) (a). Differences in regional distribution of PBpositive stains in the cortex, sub-cortex, and cerebellum of LPS- and saline-treated mice (comparisons shown in blue) (a). Representative images showing PB-positive stains in different brain regions (b). Scale bar $=100 \mu \mathrm{m}$. Significantly higher PB-positive area in the LPS- compared with saline-treated mice (c). Data are presented as mean \pm SEM. Mann-Whitney $U$ test to compare two groups and two-way repeated measure ANOVA with Bonferroni's post-test for more than two groups; ${ }^{*} p<0.05,{ }^{* *} p<0.01,{ }^{* * *} p<0.0001$

$\mathrm{CMH}$ are primarily found to be associated with a rupture in the cerebral vessel wall that allows extravasation of $\mathrm{RBC}$ into the brain parenchyma [28]. In our model, LPS-induced $\mathrm{CMH}$ were significantly associated with endothelial activation (determined by measuring ICAM1 immunoreactivity) and BBB disruption (determined by measuring brain IgG immunoreactivity). Further, larger $\mathrm{CMH}$ were associated with a larger BBB disruption. LPS can trigger an inflammatory response within the brain parenchyma, and studies show that neuroinflammation is associated with microvascular injury and microhemorrhage development $[29,30]$. In the present study, endothelial damage along with neuroinflammation (evident from the increase in microglia/macrophage- and astrocyte-positive immunoreactivity in LPS-treated mice) were significantly associated with $\mathrm{CMH}$ development, indicating a role for BBB injury and neuroinflammation in the pathogenesis of LPS-induced $\mathrm{CMH}$.

In the current study, LPS-induced $\mathrm{CMH}$ developed at the level of both the cerebral capillaries (vessel diameter $<10 \mu \mathrm{m}$ ) [31] and larger vessels (vessel diameter $>10 \mu \mathrm{m}$ ) [32]. The majority of the vessels associated with LPS-induced CMH were $<10 \mu \mathrm{m}$ in diameter, suggesting that brain capillaries [31] are more susceptible to LPSinduced $\mathrm{CMH}$ development in this model. Our finding of a capillary involvement in $\mathrm{CMH}$ development is consistent with recent rodent studies in which hypertensionand hypoxia-induced $\mathrm{CMH}$ were also localized around cerebral microvessels [5, 33].

Existing mouse models of $\mathrm{CMH}$ include mice that over-express mutant amyloid precursor protein to study cerebral amyloid angiopathy (CAA)-associated $\mathrm{CMH}$ [2], hypertensive mice [5] to study hypertension-associated $\mathrm{CMH}$, and the hypoxia-reoxygenation-induced $\mathrm{CMH}$ mouse model to study high-altitude-associated $\mathrm{CMH}$ [33]. In the CAA mouse model, mice spontaneously develop $\mathrm{PB}$-positive lesions by 15-24 months of age [2] and development of $\mathrm{CMH}$ can be exacerbated by passive immunization $[3,34]$ or by inducing bilateral common carotid artery stenosis [4]. Two-photon-excited microscopy has been used to induce localized cortical $\mathrm{CMH}$ [35], and hyperhomocysteinemia (HHcy) has also been 
Table 1 Correlation between ICAM-1-, IgG-, Iba1-, and GFAP-positive area with the (a) number of $\mathrm{CMH}$, (b) $\mathrm{CMH}$ size, and (c) $\mathrm{CMH}$-positive area in the 2-day experiment

\begin{tabular}{|c|c|c|c|c|}
\hline & $\begin{array}{l}\text { ICAM-1-positive area (\%) } \\
r\end{array}$ & $\begin{array}{l}\text { IgG-positive area (\%) } \\
r\end{array}$ & $\begin{array}{l}\text { Iba1-positive area (\%) } \\
r\end{array}$ & $\begin{array}{l}\text { GFAP-positive area (\%) } \\
r\end{array}$ \\
\hline No. of $\mathrm{CMH} /$ section & $0.86^{* * *}$ & 0.38 & $0.85^{* * *}$ & $0.83^{* *}$ \\
\hline $\mathrm{CMH}$ size $\left(\mu \mathrm{m}^{2}\right)$ & 0.31 & $0.56^{*}$ & $0.72^{* *}$ & $0.62^{*}$ \\
\hline CMH-positive area (\% total area) & $0.70^{* *}$ & $0.53^{*}$ & $0.88^{* * *}$ & $0.85^{* * *}$ \\
\hline
\end{tabular}

One-tailed $p$ values: ${ }^{*} p<0.05,{ }^{* *} p<0.01,{ }^{* * *} p<0.001$

A

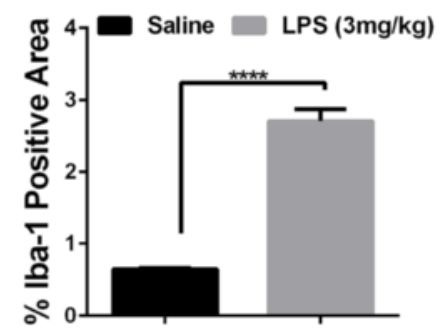

B Saline
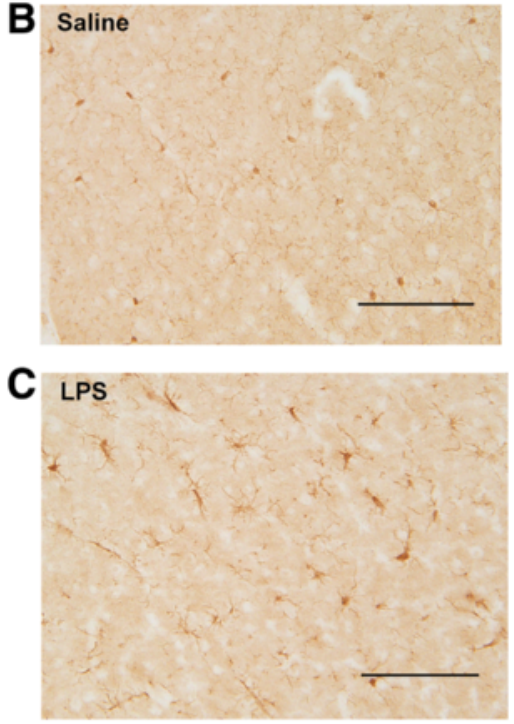

D

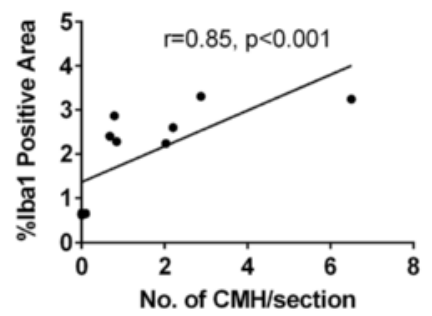

GFAP
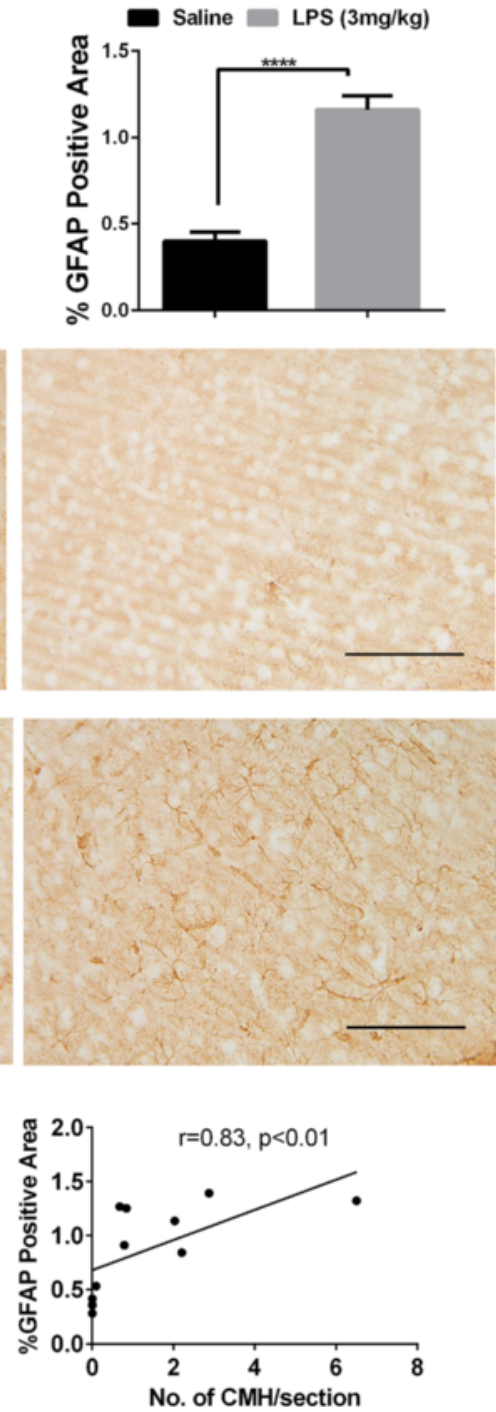

Fig. 5 Neuroinflammation and CMH development: Significantly higher Ibal (left panel)- and GFAP (right panel)-positive immunoreactive area in the LPS-treated mice $(n=7)(3 \mathrm{mg} / \mathrm{kg}$, at 0, 6, and $24 \mathrm{~h})$ compared with saline controls $(n=4)(\mathbf{a})$ in the 2-day model. Representative images showing Ibal (left)- and GFAP (right)-positive immunoreactive area in the saline (b)- and LPS (c)-treated mice (scale bar $=50 \mu$ m; images were taken at $\times 20$ ). The $\mathrm{CMH}$ number was significantly associated with both Iba1 (left)- and GFAP (right)-positive immunoreactive area in the 2-day model (3 mg/kg, at 0, 6, and 24 h) (d). Data are presented as mean \pm SEM. Student's $t$ test to compare two groups; ${ }^{* * *} p<0.0001$; one-tailed Spearman rho correlation for correlation analysis 

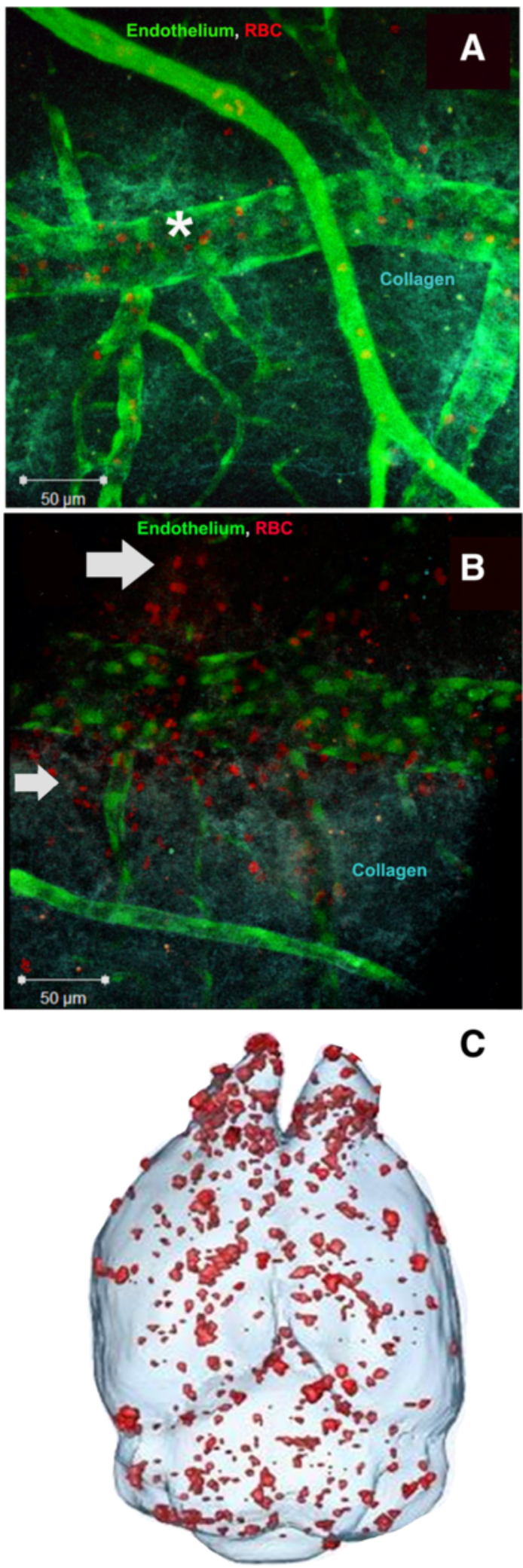

Fig. 6 Ex vivo fluorescence and second-harmonic generation imaging showing SHG of collagen (blue color), fluorescence of GFP in endothelial cells (green), and PKH26 fluorescence marker in red blood cells (red) in the brains of saline (a) and triple-dose $(3 \mathrm{mg} / \mathrm{kg}$ at 0,6 , and 24 h) LPS-treated Tie2-GFP mice sacrificed at 2 days (b). All images are 3D reconstructions in enface (or true focus) geometry. Asterisk sign indicates RBC confined within the brain vasculature in a saline mouse (a), and arrows indicate extravasation of RBC from the brain vasculature into the brain parenchyma in a LPS-treated mouse (b). Superior perspective of 3D surface renderings of brain showing distribution of cerebral microbleeds (in red) in mice from the 7-day experiment treated with a triple-dosing regimen of LPS $(1 \mathrm{mg} / \mathrm{kg}$ at 0 , 6, and 24 h) (c) using T2*-weighted 3D FLASH MRI

used to produce $\mathrm{CMH}$ [36]. One commonality between the existing mouse models of $\mathrm{CMH}$ and the present LPS-induced mouse model is inflammation. CMH development was found to be associated with an enhanced M1-type neuroinflammatory response in the CAA mouse model, with increased microglial activation and brain cytokine levels in the HHcy mouse model, and a rapid inflammatory response up to $200 \mu \mathrm{m}$ from $\mathrm{CMH}$ in the two-photon-excited microscopy mouse model. These observations are consistent with the current model where we observed a significant association between markers of neuroinflammation (microglia/ macrophage and astrocytes) and $\mathrm{CMH}$ development.

In the existing rodent models $[2,3,34,36]$ and clinically [6], PB staining for hemosiderin is commonly used to detect old $\mathrm{CMH}$ in post mortem brain tissues. Further, the current standardized method used to visualize cerebral microbleeds in humans is MRI, which relies on the paramagnetic properties of hemosiderin. Detection of hemosiderin in brain tissue is thus crucial to develop a clinically relevant mouse model of cerebral microbleeds. PB-positive lesions cannot be detected 2 days after LPS injection [15], and we thus modified the dosing regimen and extended our study to 7 days to detect PBpositive lesions and cerebral microbleeds using MRI in our model. C57BL/6 mice treated with a triple intraperitoneal dose of LPS $(1 \mathrm{mg} / \mathrm{kg}$ at 0,6 , and $24 \mathrm{~h})$ showed robust development of PB-positive lesions at 7 days with no mortality. Visualization of LPS-induced $\mathrm{CMH}$ at 7 days using MRI demonstrates and confirms radiologically the presence of cerebral microbleeds in the current model.

Clinically, typical $\mathrm{CMH}$ location is associated with distinct underlying vascular pathologies: hypertensive vasculopathy is associated with $\mathrm{CMH}$ that are located in the basal ganglia, thalamus, cerebellum, and brain stem, while CAA is associated with lobar $\mathrm{CMH}$ [1]. Varied neuroanatomical $\mathrm{CMH}$ distribution is also observed in hypoxia-reoxygenation- and hypertension-induced $\mathrm{CMH}$ mouse models; however, $\mathrm{CMH}$ density in these models is higher in the cortex or the olfactory bulb $[5,33]$. In 
the current LPS model, we saw widespread distribution of both H\&E- and PB-positive lesions. The regional distribution patterns of both $\mathrm{H} \& \mathrm{E}-$ and $\mathrm{PB}$-positive lesions were similar with maximum $\mathrm{CMH}$ density in the cerebellum and the brain stem region followed by the cerebrum (sub-cortex and cortex). Surface $\mathrm{CMH}$ that were grossly visible on the brain surface post-LPS treatment were also significantly higher in the cerebellum and brain stem region. The presence of surface $\mathrm{CMH}$ suggests a phenomenon similar to cortical superficial siderosis [37], and more extensive analysis of these lesions by MRI will clarify whether the processes are fundamentally similar. Though the mechanisms underlying the observed regional distribution pattern of $\mathrm{CMH}$ in the current study are not clear, higher distribution in the cerebellum most likely relates to the vulnerability of cerebellum blood vessels to inflammation.

\section{Conclusions}

Compared to the existing mouse models of $\mathrm{CMH}$, the current model appears to offer considerable advantages. The LPS-induced mouse model is inflammation-based (not limited to CAA-, hypoxic brain injury-, or hypertension-induced $\mathrm{CMH}$ ), non-invasive, and shows rapid ( 2 and 7 days) development of $\mathrm{CMH}$ with negligible mortality. Our work shows that LPS-induced $\mathrm{CMH}$ are primarily associated with cerebral capillaries, and $\mathrm{BBB}$ dysfunction and neuroinflammation play a role in $\mathrm{CMH}$ development in this model. Additionally, the cerebellum/brain stem region is more susceptible to LPS-induced $\mathrm{CMH}$ development. The ease of development of both H\&E- and PB-positive CMH, and MRIvisible cerebral microbleeds makes the LPS-induced mouse model suitable to study the pathophysiology of inflammation-induced $\mathrm{CMH}$.

\section{Acknowledgements}

We would like to thank Kelley Kilday, Alexis Vasquez, Quoc Tuan Pham Ngo, Gurjit Pannu, Arash Jafari, Kristie P Nguyen, and Natalie Hoi Yan Chan for their assistance.

\section{Funding}

Funding for this study was provided by a grant from NINDS: RO1 NSO20989 (MJF and DHC) and P41EB015890 from the National Institute of Biomedical Imaging and Bioengineering (TBK).

\section{Availability of data and materials}

Community-established norms of data sharing are fully respected.

\section{Authors' contributions}

RKS designed and performed the experiments, analyzed the data, prepared the figures, and wrote the paper. MMG performed the experiments and collected the data. W performed the Tie-2 GFP experiments and immunohistochemical staining. TBK performed the two-photon-excited fluorescence microscopy. MS performed the mouse brain MRI. AKD helped with the mouse brain sectioning. APH helped with the data analysis. RK provided expertise with the mouse brain histology. DHC and MJF participated in the conception of the study and have been involved in revising the manuscript critically for important intellectual content. MJF conceived the study, designed and coordinated the experiments, and helped in the drafting and editing of the manuscript. All authors read and approved the final manuscript.

\section{Competing interests}

Mark J. Fisher has received support from Boehringer-Ingelheim and Otsuka Pharmaceutical Company (research grants).

Consent for publication

Not applicable.

\section{Ethics approval and consent to participate}

All animal procedures were approved by the UCI Institutional Animal Care and Use Committee and were carried out in compliance with the University Laboratory Animal Resources regulations. This work does not involve any applicable consent to participate.

\section{Author details}

'Department of Biopharmaceutical Sciences, School of Pharmacy, Keck Graduate Institute, Claremont, CA, USA. ${ }^{2}$ Department of Neurology, University of California, Irvine, CA, USA. Institute for Memory Impairments and Neurological Disorders, University of California, Irvine, CA, USA. ${ }^{4}$ Beckman Laser Institute, University of California, Irvine, CA, USA. ${ }^{5}$ Department of Radiology, University of California, San Diego, CA, USA. ${ }^{6}$ Department of Pathology and Laboratory Medicine, University of California, Irvine, CA, USA ${ }^{7}$ Department of Anatomy and Neurobiology, University of California, Irvine, CA, USA. ${ }^{8}$ UC Irvine Medical Center, 101 The City Drive South, Shanbrom Hall, Room 121, Orange, CA 92868, USA.

Received: 6 April 2016 Accepted: 20 August 2016

Published online: 30 August 2016

\section{References}

1. Greenberg SM, Vernooij MW, Cordonnier C, Viswanathan A, Al-Shahi Salman $\mathrm{R}$, Warach S, et al. Cerebral microbleeds: a guide to detection and interpretation. Lancet Neurol. 2009:8(2):165-74. Epub 2009/01/24.

2. Fisher M, Vasilevko V, Passos GF, Ventura C, Quiring D, Cribbs DH. Therapeutic modulation of cerebral microhemorrhage in a mouse model of cerebral amyloid angiopathy. Stroke. 2011;42(11):3300-3. Epub 2011/09/10.

3. Meyer-Luehmann M, Mora JR, Mielke M, Spires-Jones TL, de Calignon A, von Andrian UH, et al. T cell mediated cerebral hemorrhages and microhemorrhages during passive Abeta immunization in APPPS1 transgenic mice. Mol Neurodegener. 2011;6:22. Epub 2011/03/11.

4. Okamoto Y, Yamamoto T, Kalaria RN, Senzaki H, Maki T, Hase Y, et al. Cerebral hypoperfusion accelerates cerebral amyloid angiopathy and promotes cortical microinfarcts. Acta Neuropathol. 2012;123(3):381-94. Epub 2011/12/16

5. Toth P, Tarantini S, Springo Z, Tucsek Z, Gautam T, Giles CB, et al. Aging exacerbates hypertension-induced cerebral microhemorrhages in mice: role of resveratrol treatment in vasoprotection. Aging Cell. 2015;14(3):400-8. Epub 2015/02/14.

6. Fisher M, French S, Ji P, Kim RC. Cerebral microbleeds in the elderly: a pathological analysis. Stroke. 2010;41(12):2782-5. Epub 2010/10/30.

7. Correa DG, Cruz Junior LC, Bahia PR, Gasparetto EL. Intracerebral microbleeds in sepsis: susceptibility-weighted MR imaging findings. Arq Neuropsiquiatr. 2012;70(11):903-4. Epub 2012/11/24.

8. Yamashiro K, Tanaka R, Hoshino Y, Hatano T, Nishioka K, Hattori N. The prevalence and risk factors of cerebral microbleeds in patients with Parkinson's disease. Parkinsonism Relat Disord. 2015;21(9):1076-81. Epub 2015/07/05

9. Lahousse L, Vernooij MW, Darweesh SK, Akoudad S, Loth DW, Joos GF, et al. Chronic obstructive pulmonary disease and cerebral microbleeds. The Rotterdam Study. Am J Respir Crit Care Med. 2013;188(7):783-8. Epub 2013/07/28

10. Huang YL, Kuo YS, Tseng YC, Chen DY, Chiu WT, Chen CJ. Susceptibilityweighted MRI in mild traumatic brain injury. Neurology. 2015;84(6):580-5. Epub 2015/01/13

11. Franceschi C, Campisi J. Chronic inflammation (inflammaging) and its potential contribution to age-associated diseases. J Gerontol A Biol Sci Med Sci. 2014;69 Suppl 1:S4-9. Epub 2014/05/17. 
12. Miwa K, Tanaka M, Okazaki S, Furukado S, Sakaguchi M, Kitagawa K. Relations of blood inflammatory marker levels with cerebral microbleeds. Stroke. 2011;42(11):3202-6. Epub 2011/08/27.

13. Shoamanesh A, Preis SR, Beiser AS, Vasan RS, Benjamin EJ, Kase CS, et al. Inflammatory biomarkers, cerebral microbleeds, and small vessel disease: Framingham Heart Study. Neurology. 2015;84(8):825-32. Epub 2015/01/30.

14. Romero JR, Preis SR, Beiser AS, DeCarli C, Lee DY, Viswanathan A, et al. Lipoprotein phospholipase A2 and cerebral microbleeds in the Framingham Heart Study. Stroke. 2012;43(11):3091-4. Epub 2012/09/11.

15. Liu S, Grigoryan MM, Vasilevko V, Sumbria RK, Paganini-Hill A, Cribbs DH, et al. Comparative analysis of H\&E and Prussian blue staining in a mouse model of cerebral microbleeds. J Histochem Cytochem. 2014;62(11):767-73. Epub 2014/07/27

16. Dziedzic T. Systemic inflammation as a therapeutic target in acute ischemic stroke. Expert Rev Neurother. 2015;15(5):523-31. Epub 2015/04/14.

17. Hallenbeck JM, Dutka AJ, Kochanek PM, Siren A, Pezeshkpour GH, Feuerstein G. Stroke risk factors prepare rat brainstem tissues for modified local Shwartzman reaction. Stroke. 1988;19(7):863-9. Epub 1988/07/01.

18. Rouhl RP, Damoiseaux JG, Lodder J, Theunissen RO, Knottnerus IL, Staals J, et al. Vascular inflammation in cerebral small vessel disease. Neurobiol Aging. 2012;33(8):1800-6. Epub 2011/05/24.

19. Bosmann M, Ward PA. The inflammatory response in sepsis. Trends Immunol. 2013;34(3):129-36. Epub 2012/10/06

20. Pauletto $P$, Rattazzi M. Inflammation and hypertension: the search for a link. Nephrol Dial Transplant. 2006;21(4):850-3. Epub 2006/02/09.

21. Abbott NJ. Inflammatory mediators and modulation of blood-brain barrier permeability. Cell Mol Neurobiol. 2000;20(2):131-47. Epub 2000/03/04

22. Fisher M. Cerebral microbleeds: where are we now? Neurology. 2014;83(15): 1304-5. Epub 2014/09/05.

23. Juskewitch JE, Knudsen BE, Platt JL, Nath KA, Knutson KL, Brunn GJ, et al. LPS-induced murine systemic inflammation is driven by parenchymal cell activation and exclusively predicted by early MCP-1 plasma levels. Am J Pathol. 2012;180(1):32-40. Epub 2011/11/10

24. Banks WA, Gray AM, Erickson MA, Salameh TS, Damodarasamy M, Sheibani N, et al. Lipopolysaccharide-induced blood-brain barrier disruption: roles of cyclooxygenase, oxidative stress, neuroinflammation, and elements of the neurovascular unit. J Neuroinflammation. 2015; 12(1):223. Epub 2015/11/27

25. Munshi N, Fernandis AZ, Cherla RP, Park IW, Ganju RK. Lipopolysaccharideinduced apoptosis of endothelial cells and its inhibition by vascular endothelial growth factor. J Immunol. 2002;168(11):5860-6. Epub 2002/05/23.

26. Karahashi H, Michelsen KS, Arditi M. Lipopolysaccharide-induced apoptosis in transformed bovine brain endothelial cells and human dermal microvessel endothelial cells: the role of JNK. J Immunol. 2009;182(11):7280-6. Epub 2009/05/21.

27. Veszelka S, Urbanyi Z, Pazmany T, Nemeth L, Obal I, Dung NT, et al. Human serum amyloid $\mathrm{P}$ component attenuates the bacterial lipopolysaccharideinduced increase in blood-brain barrier permeability in mice. Neurosci Lett. 2003:352(1):57-60. Epub 2003/11/15.

28. Poels MM, Ikram MA, van der Lugt A, Hofman A, Niessen WJ, Krestin $G P$, et al. Cerebral microbleeds are associated with worse cognitive function: the Rotterdam Scan Study. Neurology. 2012;78(5):326-33. Epub 2012/01/21.

29. Glushakova OY, Johnson D, Hayes RL. Delayed increases in microvascular pathology after experimental traumatic brain injury are associated with prolonged inflammation, blood-brain barrier disruption, and progressive white matter damage. J Neurotrauma. 2014;31(13):1180-93. Epub 2014/02/26.

30. He XF, Lan Y, Zhang Q, Liu DX, Wang Q, Liang FY, et al. Deferoxamine inhibits microglial activation, attenuates blood-brain barrier disruption, rescues dendritic damage and improves spatial memory in a mouse model of microhemorrhages. J Neurochem. 2016. Epub 2016/05/12.

31. Stefanovic B, Hutchinson E, Yakovleva V, Schram V, Russell JT, Belluscio L, et al. Functional reactivity of cerebral capillaries. J Cereb Blood Flow Metab. 2008;28(5):961-72. Epub 2007/12/07.

32. Santisakultarm TP, Cornelius NR, Nishimura N, Schafer Al, Silver RT, Doerschuk PC, et al. In vivo two-photon excited fluorescence microscopy reveals cardiac- and respiration-dependent pulsatile blood flow in cortical blood vessels in mice. Am J Physiol Heart Circ Physiol. 2012;302(7):H1367-77. Epub 2012/01/24.

33. Hoffmann A, Kunze R, Helluy X, Milford D, Heiland S, Bendszus M, et al. High-field MRI reveals a drastic increase of hypoxia-induced microhemorrhages upon tissue reoxygenation in the mouse brain with strong predominance in the olfactory bulb. PLoS One. 2016;11(2):e0148441. Epub 2016/02/11

34. Wilcock DM, Colton CA. Immunotherapy, vascular pathology, and microhemorrhages in transgenic mice. CNS Neurol Disord Drug Targets. 2009;8(1):50-64. Epub 2009/03/12.

35. Rosidi NL, Zhou J, Pattanaik S, Wang P, Jin W, Brophy M, et al. Cortical microhemorrhages cause local inflammation but do not trigger widespread dendrite degeneration. PLoS One. 2011;6(10):e26612. Epub 2011/10/27.

36. Sudduth TL, Powell DK, Smith CD, Greenstein A, Wilcock DM. Induction of hyperhomocysteinemia models vascular dementia by induction of cerebral microhemorrhages and neuroinflammation. J Cereb Blood Flow Metab. 2013;33(5):708-15. Epub 2013/01/31.

37. Charidimou A, Linn J, Vernooij MW, Opherk C, Akoudad S, Baron JC, et al. Cortical superficial siderosis: detection and clinical significance in cerebral amyloid angiopathy and related conditions. Brain. 2015;138(Pt 8):2126-39. Epub 2015/06/28.

\section{Submit your next manuscript to BioMed Central and we will help you at every step:}

- We accept pre-submission inquiries

- Our selector tool helps you to find the most relevant journal

- We provide round the clock customer support

- Convenient online submission

- Thorough peer review

- Inclusion in PubMed and all major indexing services

- Maximum visibility for your research

Submit your manuscript at www.biomedcentral.com/submit
Biomed Central 